Article

\title{
Estimation of Nucleon D-Term in QCD
}

\author{
Igor V. Anikin
}

Bogoliubov Laboratory of Theoretical Physics, JINR, 141980 Dubna, Russia; anikin@theor.jinr.ru

Received: 12 April 2019; Accepted: 27 June 2019; Published: 28 June 2019

\begin{abstract}
Using the light-cone sum rules at leading order, we present an approach to perform the preliminary upper estimation for the nucleon gravitational form factor $\mathbb{D}(t)$ ( $D$-term contribution). Comparison with the experimental data and with the results of different models is discussed.
\end{abstract}

Keywords: gravitational form factors; QCD; electromagnetic form factors; light-cone sum rules; $D$-term

PACS: $12.38 .-t ; 14.20 . D h ; 13.40 . G p$

\section{Introduction}

It is well-known that the hadron matrix element of energy-momentum tensor (EMT) can provide information on fundamental characteristics of particles such as mass and spin [1-5]. One of the EMT form factors has been related to the D-term [6] which can be called as the last unknown fundamental hadron characteristic determining the spatial deformations as well as defining the mechanical properties of hadrons [1-3]. The analogy of $D$-term with the vacuum cosmological constant has been studed in [7]. The $D$-term has also been calculated in the framework of the dispersion relation approach where the good agreement with the chiral quark-soliton model has been achieved [8]. Recently, the results of [2] have been extended to the different frames where the nucleon has the non-vanishing average momentum [9].

Since the energy-momentum tensor is the interplay between gravitation as an external field and the matter fields (in full analogy with the gauge fields which interact with particles through the corresponding electromagnetic current), we adopt the light-cone sum rules (LCSRs) developed for different nucleon electromagnetic form factors in [10-12].

In the present paper, we develop the light-cone sum rules for the preliminary upper estimation $M$ of the nucleon gravitational form factor $\mathbb{D}(t),|\mathbb{D}(t)| \leq M$. Unfortunately, the full information on the gravitation form factor $\mathbb{D}(t)$ ( $D$-term form factor) cannot be obtained within the LCSRs due to the lack of the sum rules that use the minus and perpendicular light-cone projections of electromagnetic current. To estimate $\mathbb{D}(t)$, we study the valence quark contributions in nucleon that originate from the leading collinear twist-2 combination of the electromagnetic current. In order to approach the region of small $t$, where the LCSRs approach is really useless, we first approximate the form factor derived for the region of large $t$ by the appropriate multipole function and, then, we do the extrapolation of fitted form factor to the region of small $t$. Our final preliminary prediction demonstrates reasonably good agreement with the first experimental data $[13,14]$ together with the chiral quark-soliton and Skyrme model results for $D$-term contributions [2].

\section{Energy-Momentum Tensor}

For the quark contribution, the Belinfante improved energy-momentum tensor is nothing but the local geometrical twist-2 operator which reads 


$$
\Theta_{q}^{\mu v}(0)=\frac{i}{2} \mathcal{R}_{\tau=2}^{\mu v}
$$

and it can be expressed through the non-local operator as (cf. [15])

$$
\begin{aligned}
-2 i \Theta_{q}^{\mu v}(0) & =\lim _{y \rightarrow 0} \frac{\partial}{\partial y_{v}} \int_{0}^{1} d u \frac{\partial}{\partial y_{\mu}}\left[\bar{\psi}(0) \hat{y}[0 ; u y]_{A} \psi(u y)\right] \\
& -(\text { trace) }
\end{aligned}
$$

where $y=\left(y^{+}, y^{-}, \overrightarrow{\mathbf{y}}_{\perp}\right)$. From now on, we do not show the trace subtractions. In the paper, we are restricted by the bilinear quark combinations with the spin projection $s_{a}=+1$. Hence, we deal with the collinear twist-2 quark combination $\left[\bar{\psi}_{+} \psi_{+}\right]$of $\mathcal{R}_{\tau=2}^{\mu \nu}$. In this connection, we also introduce the local geometrical twist-2 operator which reads

$$
\widetilde{\mathcal{R}}_{\tau=2}^{\mu v}=\lim _{y \rightarrow 0} \frac{\partial}{\partial y_{v}} \int_{0}^{1} d u \frac{\partial}{\partial y_{\mu}}\left[\bar{\psi}(0) y_{\alpha} \gamma^{\alpha,+}[0 ; u y]_{A} \psi(u y)\right] .
$$

Notice that this operator actually differs from the operator $\mathcal{R}_{\tau=2}^{\mu \nu}$ but $\widetilde{\mathcal{R}}_{\tau=2}^{++}=\mathcal{R}_{\tau=2}^{++}$.

Moreover, the operator $\mathcal{R}_{\tau=2}^{+-}$can be written as

$$
\begin{aligned}
\mathcal{R}_{\tau=2}^{+-} & =\frac{1}{2}\left(\bar{\psi}(0) \gamma^{+} \overrightarrow{\mathcal{D}}^{-} \psi(0)+\bar{\psi}(0) \gamma^{-} \overrightarrow{\mathcal{D}}^{+} \psi(0)\right) \\
& =\widetilde{\mathcal{R}}_{\tau=2}^{+-}+\frac{1}{2} \bar{\psi}(0) \gamma^{-} \overrightarrow{\mathcal{D}}^{+} \psi(0),
\end{aligned}
$$

where the first term with the quark combination involving $\left[\bar{\psi}_{+} \psi_{+}\right]$defines $\widetilde{\mathcal{R}}_{\tau=2}^{+-}$and the second term with $\left[\bar{\psi}_{-} \psi_{-}\right]$is kept intact because it is beyond the direct computations within our approach. Despite, the $\left[\bar{\psi}_{-} \psi_{-}\right]$contribution to the projection $\Theta^{+-}$, which we eventually need, remains unavailable for the explicit LCSRs calculations, we however can still make some reasonable estimation for this kind of contribution.

\section{Gravitational Form Factors}

We now remind the standard definitions for the relevant gravitational form factors. The quark contribution to the hadron matrix element of the Belinfanté improved energy-momentum tensor operator reads (see, for instance, [3])

$$
\begin{aligned}
& \left\langle P^{\prime}\left|\Theta_{\mu v}^{(q)}(0)\right| P\right\rangle=\bar{N}\left(P^{\prime}\right)\left[\mathbb{A}(t) \frac{\bar{P}_{\mu} \bar{P}_{v}}{m_{N}}+i \mathrm{~J}(t) \frac{\bar{P}_{\{\mu} \sigma_{v\}}}{m_{N}}+\right. \\
& \left.\mathbb{D}(t) \frac{\Delta_{\mu} \Delta_{v}-g_{\mu v} \Delta^{2}}{4 m_{N}}+g_{\mu v} m_{N} \overline{\mathbb{C}}(t)\right] N(P),
\end{aligned}
$$

where

$$
\begin{aligned}
& \mathrm{J}(t)=\frac{1}{2}(\mathbb{A}(t)+\mathbb{B}(t)), \quad a_{\{\mu} b_{v\}}=\frac{1}{2}\left(a_{\mu} b_{v}+a_{\nu} b_{\mu}\right), \\
& \bar{P}=\frac{1}{2}\left(P^{\prime}+P\right), \quad \Delta=P^{\prime}-P, \quad \Delta^{2}=-t .
\end{aligned}
$$


Notice that the Belinfanté improved energy-momentum or angular momentum tensor includes the contribution from the spin momentum tensor. As usual, the hadron momenta can be expanded as [10]

$$
P=p+\frac{m_{N}^{2}}{2} n, \quad \Delta=P \cdot \Delta n+\Delta_{\perp} .
$$

To calculate the form factor $\mathbb{D}(t)$ we have to concentrate on a projection $\mathcal{R}_{\tau=2}^{+-}$. Since our exact computations are restricted by the consideration of $\left[\bar{\psi}_{+} \psi_{+}\right]$combination, we are dealing with the corresponding projections of $\widetilde{\mathcal{R}}_{\tau=2}^{\mu v}$ rather than the projections of $\mathcal{R}_{\tau=2}^{\mu v}$. With respect to the $\left[\bar{\psi}_{-} \psi_{-}\right]$ combination, we propose a reliable recipe for estimations of this contribution.

In analogy with the nucleon electromagnetic form factors [10-12], we define the amplitude which corresponds to the hadron matrix element of the energy-momentum tensor as

$$
\begin{aligned}
& T_{\left[\bar{\psi}+\psi_{+}\right]}^{\mu v}(P, \Delta) \equiv\left\langle P^{\prime}\left|\widetilde{\mathcal{R}}_{\tau=2}^{\mu v}\right| P\right\rangle=\lim _{y \rightarrow 0} \frac{\partial}{\partial y_{v}} \int_{0}^{1} d u \frac{\partial}{\partial w_{\mu}} \int\left(d^{4} z\right) e^{-i \Delta \cdot z}\langle 0| T \eta(0) \\
& \times\left[\bar{\psi}(w+z) w^{\alpha} \gamma^{\alpha,+}[w+z ;-w+z]_{A} \psi(-w+z)\right]|P\rangle,
\end{aligned}
$$

where $w=u y \cdot \eta(0)$ stands for the Ioffe interpolation current defined as

$$
\eta(0)=\varepsilon^{i j k}\left[u^{i}(0) C \gamma_{\alpha} u^{j}(0)\right] \gamma_{5} \gamma_{\alpha} d^{k}(0) .
$$

Below, the colour indices $i, j, k, .$. are omitted.

By the direct calculation of (8), using the distribution amplitude parametrization (see for example [10]), one concludes that in order to extend the preceding calculations performed for electromagnetic form factors to the case of gravitational form factors we have to weight the electromagnetic form factors with the certain tensor structure. So, the exact expression for the amplitude for $d$-quark contribution is given by

$$
T_{\left[\bar{\psi}_{+} \psi_{+}\right]}^{\mu v(d)}(P, \Delta)=\frac{1}{8} \int \mathcal{D} x_{i}\left(2 p_{\mu}\left[2 x_{1} P+\Delta\right]_{v}+(\mu \leftrightarrow v)\right) T_{e m}^{(d)+}\left(x_{i} ; P, \Delta\right),
$$

where

$$
T_{e m}^{(q)+}\left(x_{i} ; P, \Delta\right)=\left\{m_{N} \mathcal{A}_{e m}^{+(q)}\left(x_{i} ; \Delta^{2}, P^{\prime 2}\right)+\hat{\Delta}_{\perp} \mathcal{B}_{e m}^{+(q)}\left(x_{i} ; \Delta^{2}, P^{\prime 2}\right)\right\} N^{+}(P),
$$

and $\mathcal{A}_{e m}^{+(q)}, \mathcal{B}_{e m}^{+(q)}$ have been taken from [10]. In a similar manner we can write down the $u$-quark contribution.

From Equation (10), we conclude that the tensor structure of the amplitude (8) is entirely determined by the tensor

$$
2 p_{\mu}\left[2 x_{i} P+\Delta\right]_{v}+(\mu \leftrightarrow v) .
$$

Since the Ioffe current $\eta(0)$ has been used as the interpolation current, we focus on the valence quark contributions to nucleon. Notice that all amplitudes and form factors should be understood as the objects where the summation over $u$ - and $d$-flavours have been implemented: $T_{\mu v}=T_{\mu \nu}^{(u)}+T_{\mu \nu}^{(d)}$ etc. 


\section{Estimation of the Form Factor $\mathbb{D}(t)$}

We are now going over to the plus-minus light-cone projection of the amplitude which is $T^{+-}(P, \Delta)$. For the sake of shortness, we introduce the combination given by

$$
\mathbb{F}(t)=2 m_{N}\left(m_{N} \overline{\mathbb{C}}(t)-\frac{\Delta_{\perp}^{2}}{4 m_{N}} \mathbb{D}(t)\right)
$$

For our goal, we first focus on the $T_{1}$-type (or $\mathcal{A}$-type) of amplitudes, we have the following relations (for the LCSR notations, see [10])

$$
\begin{aligned}
& \frac{\lambda_{1} m_{N}}{m_{N}^{2}-P^{\prime 2}}\left(m_{N}^{2} \mathbb{A}(t)+\left[\frac{\Delta_{\perp}^{2}}{4}+P \cdot \Delta\right] \mathbb{A}(t)+\frac{\Delta_{\perp}^{2}}{4} \mathbb{B}(t)\right. \\
& +\mathbb{F}(t)) N^{+}(P)=m_{N}\left(\bar{T}_{1\left[\bar{\psi}_{+} \psi_{+}\right]}^{+}+\bar{T}_{1\left[\bar{\psi}_{-} \psi_{-}\right]}^{+-}\right) N^{+}(P),
\end{aligned}
$$

where $\mathbb{A}(t)$ and $\mathbb{B}(t)$ can be expressed through the plus-plus projection of the amplitudes, $\mathcal{A}^{++}$and $\mathcal{B}^{++}$respectively, and

$$
\begin{aligned}
& m_{N}\left(\bar{T}_{1\left[\bar{\psi}_{+} \psi_{+}\right]}^{+}+\bar{T}_{1\left[\bar{\psi}-\psi_{-}\right]}^{+-}\right)=m_{N} \bar{T}_{1\left[\bar{\psi}_{-} \psi_{-}\right]}^{+-}+ \\
& \frac{m_{N}}{\pi} \int_{0}^{s_{0}} \frac{d s}{s-P^{\prime 2}} \operatorname{Im}\left\{\frac{m_{N}^{2}}{4} \mathcal{A}^{++}(s, t)+\frac{P \cdot \Delta}{4} \mathcal{A}_{e m}^{+}(s, t)\right\} .
\end{aligned}
$$

Notice that unlike the plus-plus light-cone projection, the Equation (14) do not form the closed system of equations. Hence, there is no way to compute the form factors $\mathbb{D}(t)$ directly. Instead, we shall try to estimate the form factor $\mathbb{D}(t)$.

The next step is to perform the Borel transforms of Equation (14). Afterwards, we derive the first representation for $\mathbb{F}(t)$ which reads

$$
\begin{aligned}
& \mathbb{F}(t)=T_{1\left[\bar{\psi}-\psi_{-}\right]}^{+-}-\int \hat{d} \mu(s)\left\{\frac{m_{N}^{2}}{4} \mathcal{A}^{++}-\right. \\
& \left.\frac{\Delta_{\perp}^{2}}{4}\left(\frac{1}{2} \mathcal{A}^{++}+\mathcal{B}^{++}-\frac{1}{2} \mathcal{A}_{e m}^{+}\right)\right\},
\end{aligned}
$$

where

$$
\int \hat{d} \mu(s)\{\mathcal{F}\}=\frac{1}{\lambda_{1} \pi} \int_{0}^{s_{0}} d s e^{\left(m_{N}^{2}-s\right) / M^{2}} \operatorname{Im}\{\mathcal{F}(s, t)\}
$$

In the same manner, working with the $T_{4}$-type (or $\mathcal{B}$-type) of amplitudes, we derive the the second equivalent representation for $\mathbb{F}(t)$ which is

$$
\begin{aligned}
& \mathbb{F}(t)=2 \bar{T}_{4\left[\bar{\psi}-\psi_{-}\right]}^{+-}-\int \hat{d} \mu(s)\left\{\frac{m_{N}^{2}}{2}\left(\mathcal{A}^{++}-\mathcal{B}^{++}\right)-\right. \\
& \left.\frac{\Delta_{\perp}^{2}}{4}\left(2 \mathcal{B}^{++}-\mathcal{B}_{\text {em }}^{+}\right)\right\} .
\end{aligned}
$$

Combining (16) and (18), we obtain the relation as

$$
R(t)=\frac{4}{\Delta_{\perp}^{2}}\left\{2 \bar{T}_{4\left[\bar{\psi}_{-} \psi_{-}\right]}^{+-}-\bar{T}_{1\left[\bar{\psi}-\psi_{-}\right]}^{+-}\right\},
$$

where, by introduction, 


$$
R(t)=\int \hat{d} \mu(s)\left\{\frac{\mathcal{A}^{++}}{2}-\mathcal{B}^{++}\right\}\left(1+\frac{2 m_{N}^{2}}{\Delta_{\perp}^{2}}\right)-\int \hat{d} \mu(s)\left\{\frac{\mathcal{A}_{e m}^{+}}{2}-\mathcal{B}_{e m}^{+}\right\} .
$$

The behavior of $R$ as functions of $t$ has been presented in Figure 1. The numerical analysis, based on our work [10], shows that $R \approx 0$, therefore we are able to estimate the unknown LCSRs contributions as

$$
2 \lim _{t \rightarrow \infty} \bar{T}_{4\left[\bar{\psi}_{-} \psi_{-}\right]}^{+-} \approx \lim _{t \rightarrow \infty} \bar{T}_{1\left[\bar{\psi}-\psi_{-}\right]}^{+-} .
$$

As one can see in Figure 1, this estimation works with a good precision for $t \geq 4 \mathrm{GeV}^{2}$.

Further, since the amplitudes of $\mathcal{A}$ - and $\mathcal{B}$-types are nothing but the invariant amplitudes of $T_{1}$ and $T_{4}$-types, having focused on $\bar{T}_{1\left[\bar{\psi}-\psi_{-}\right]}^{+-}$only, we impose that

$$
\bar{T}_{1\left[\bar{\psi}_{-} \psi_{-}\right]}^{+\stackrel{t \rightarrow \infty}{\approx}} \int \hat{d} \mu(s)\left\{\frac{1}{2} \mathcal{A}_{e m}^{+}-\frac{1}{2} \mathcal{A}^{++}\left(1-\frac{2 m_{N}^{2}}{t}\right)\right\} .
$$

We are now going to analyze of the $D$-term. Using the QCD equations of motion, we can express $\overline{\mathbb{C}}(t)$ through the nucleon matrix elements of the quark-gluon operator (for the quark contribution) or the gluon-gluon operator (for the gluon contribution) [16]. Therefore, the calculation of $\overline{\mathbb{C}}(t)$ is not available at the leading order at all. Moreover, this form factor meets the condition $\sum_{a} \overline{\mathbb{C}^{a}}(t)=0$ which shows that the total (quark and gluon) energy-momentum tensor is conserved. So, at the moment, the consideration of $\overline{\mathbb{C}}(t)$ is not presented in the present paper and is postponed to the forthcoming work. Neglecting $\overline{\mathbb{C}}$-term from $\mathbb{F}$-combination at the large $t$ and using the approximation (22), we estimate the form factor $\mathbb{D}(t)$ by (see, the Equation (16))

$$
\mathbb{D}(t) \approx-\int \hat{d} \mu(s)\left\{\frac{1}{2} \mathcal{B}^{++}+\frac{m_{N}^{2}}{t} \mathcal{A}^{++}\right\} .
$$

We emphasize that the form factor $\mathbb{D}(t)$ is independent one. Equation (23) shows that the estimated $\mathbb{D}(t)$ as a function of $t$ behaves like a combination of the form factors $\mathcal{A}^{++}$and $\mathcal{B}^{++}$.

According to the strategy of [10], the gravitational form factor $\mathbb{D}(t)$ is not reachable for a region of small $t$ within LCSRs approach. However, we can do approximate fit for this form factor in the region of large $t$ and, then, perform an extrapolation of the obtained fitting functions to the region of small $t$. Finally, the preliminary upper estimation of $\mathbb{D}(t)$ can be described by (cf. [16])

$$
\begin{aligned}
& \mathbb{D}^{\mathrm{fit}}(t)=\frac{D}{(1+e t)^{n}}, \\
& D=-2.7, \quad e=0.96 \mathrm{GeV}^{-2}, \quad n=3.43 .
\end{aligned}
$$

In the region of small $t$, for all sets of LCSR-parametrizations, this approximating function matches, see Figure 2, with the first experimental data $[13,14]$ together with the results of the chiral quark-soliton and Skyrme models presented in [2,17-19]. Our function $\mathbb{D}^{\text {fit }}(t)$ lies below than the quark-soliton prediction and above than the result of Skyrme model. 


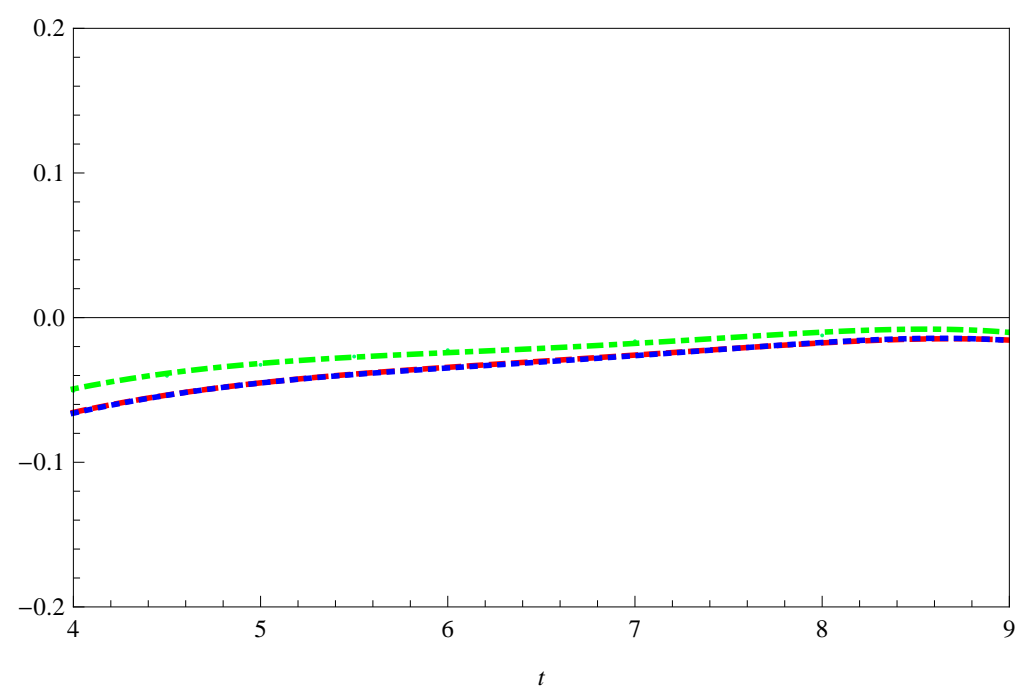

Figure 1. The functions $R(t)$. Notations: the dotted blue line corresponds to $\mathrm{ABO} 2$; the dashed red line corresponds to ABO1; the dot-dashed green line corresponds to BLW [10].

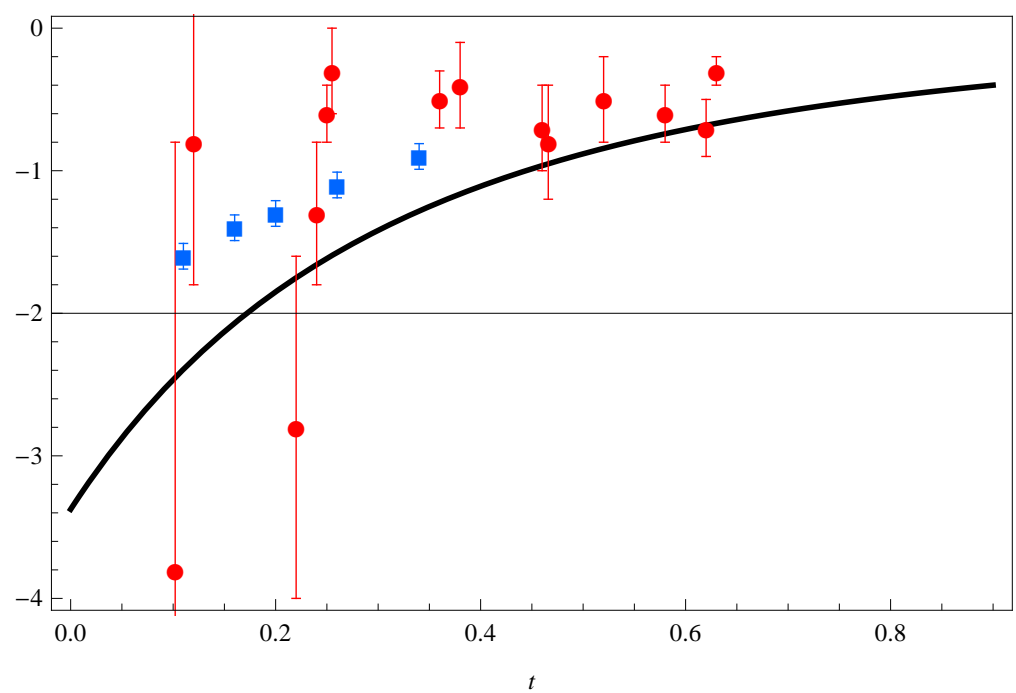

Figure 2. The preliminary upper estimation of $\frac{5}{4} \mathrm{D}^{\text {fit }}(t)$ for the region of small $t$. The experimental data on the nucleon $D$-term form factor: the blue squares from the Jefferson Lab [14], the red bullets from the lattice quantum chromodynamics (QCD) [13].

\section{Mechanical Properties of Nucleon}

Having calculated the form factor $\mathbb{D}(t)$, one computes the pressure in the center of nucleon and the hadron mechanical radius [2]. We have

$$
p_{0}=-\frac{1}{24 \pi^{2} m_{N}} \int_{0}^{\infty} d t t \sqrt{t} \mathbb{D}^{\mathrm{fit}}(t)=0.79 \mathrm{GeVfm}^{-3}
$$

for the pressure $\left[\mathrm{cf} . p_{0}^{[2]}=0.23 \mathrm{GeVfm}^{-3}\right]$, and

$$
\left\langle r^{2}\right\rangle_{\text {mech }}=6 D\left[\int_{0}^{\infty} d t \mathbb{D}^{\mathrm{fit}}(t)\right]^{-1}=13.97 \mathrm{GeV}^{-2}
$$

for the mechanical radius. 


\section{Conclusions}

The main challenge in the QCD description of any form factors is the calculation of soft contributions which correspond to the Feynman mechanism to transfer the rather large momentum. As demonstrated in many papers (see, for example, [20-23]) the LCSRs approach is attractive due to the fact that the soft contributions are calculated in terms of the same distribution amplitudes (DAs) that the perturbative QCD (pQCD) calculations include. Thus, the LCSRs can be positioned as one of the most direct relations of the different kind of form factors and hadron DAs that is available at present.

In the paper, we have presented the first preliminary estimation for the valence quark contributions to the gravitational form factor $\mathbb{D}(t)$ (the so-called $D$-term) implemented due to the LCSRs techniques at the leading order. To the region of large $t$, where the LCSRs approach can be used, we have observed that the estimated form factor $\mathbb{D}$ as a function of $t$ has the similar behaviour as the suitable combination of the well-known gravitational form factors $\mathbb{A}(t)$ and $\mathbb{B}(t)$. Regarding the small $t$ region, where the first experimental data are available $[13,14]$, the estimated $\mathbb{D}(t)$ can be approximated by the fitting multipole function $\mathbb{D}^{\text {fit }}(t)$. With the upper-estimated $\mathbb{D}^{\text {fit }}(t)$, a few quantities characterizing the so-called mechanical properties of the nucleon have been calculated. We emphasize that our result is consistent with the experimental data as well as with the results of the chiral quark-soliton and Skyrme models presented in [2,17-19].

Funding: This research received no external funding.

Acknowledgments: We are grateful to V. Braun, M. Deka, A. Manashov, M. Polyakov and P. Schweitzer for useful discussions, comments and remarks.

Conflicts of Interest: The author declares no conflict of interest.

\section{References}

1. Polyakov, M.V. Generalized parton distributions and strong forces inside nucleons and nuclei. Phys. Lett. B 2003, 555, 57. [CrossRef]

2. Polyakov, M.V.; Schweitzer, P. Forces inside hadrons: Pressure, surface tension, mechanical radius, and all that. Int. J. Mod. Phys. A 2018, 33, 1830025. [CrossRef]

3. Polyakov, M.V.; Son, H.D. Nucleon gravitational form factors from instantons: Forces between quark and gluon subsystems. arXiv 2018, arXiv:1808.00155.

4. Belitsky, A.V.; Radyushkin, A.V. Unraveling hadron structure with generalized parton distributions. Phys. Rep. 2005, 418, 1. [CrossRef]

5. Diehl, M. Generalized parton distributions. Phys. Rep. 2003, 388, 41. [CrossRef]

6. Kivel, N.; Polyakov, M.V.; Vanderhaeghen, M. DVCS on the nucleon: Study of the twist-Three effects. Phys. Rev. D 2001, 63, 114014. [CrossRef]

7. Teryaev, O. Analyticity and higher twists. Nucl. Phys. Proc. Suppl. 2013, 245, 195. [CrossRef]

8. Pasquini, B.; Polyakov, M.V.; Vanderhaeghen, M. Dispersive evaluation of the D-term form factor in deeply virtual Compton scattering. Phys. Lett. B 2014, 739, 133. [CrossRef]

9. Lorcé, C.; Moutarde, H.; Trawinski, A.P. Revisiting the mechanical properties of the nucleon. Eur. Phys. J. C 2019, 79, 89. [CrossRef]

10. Anikin, I.V.; Braun, V.M.; Offen, N. Nucleon Form Factors and Distribution Amplitudes in QCD. Phys. Rev. D 2013, 88, 114021. [CrossRef]

11. Anikin, I.V.; Braun, V.M.; Offen, N. Electroproduction of the $N^{*}(1535)$ nucleon resonance in QCD. Phys. Rev. D 2015, 92, 014018. [CrossRef]

12. Anikin, I.V.; Braun, V.M.; Offen, N. Axial form factor of the nucleon at large momentum transfers. Phys. Rev. D 2016, 94, 034011. [CrossRef]

13. Hagler, P.; Schroers, W.; Bratt, J.; Negele, J.W.; Pochinsky, A.V.; Edwards, R.G.; Richards, D.G.; Engelhardt, M.; Fleming, G.T.; Musch, B.; et al. Nucleon Generalized Parton Distributions from Full Lattice QCD. Phys. Rev. D 2008, 77, 094502. [CrossRef] 
14. Burkert, V.D.; Elouadrhiri, L.; Girod, F.X. The pressure distribution inside the proton. Nature 2018, 557, 396. [CrossRef] [PubMed]

15. Balitsky, I.I.; Braun, V.M. Evolution Equations for QCD String Operators. Nucl. Phys. B 1989, $311,541$. [CrossRef]

16. Tanaka, K. Operator relations for gravitational form factors of a spin-0 hadron. Phys. Rev. D 2018, 98, 034009. [CrossRef]

17. Goeke, K.; Grabis, J.; Ossmann, J.; Polyakov, M.V.; Schweitzer, P.; Silva, A.; Urbano, D. Nucleon form-factors of the energy momentum tensor in the chiral quark-soliton model. Phys. Rev. D 2007 75, 094021. [CrossRef]

18. Goeke, K.; Grabis, J.; Ossmann, J.; Schweitzer, P.; Silva, A.; Urbano, D. The pion mass dependence of the nucleon form-factors of the energy momentum tensor in the chiral quark-soliton model. Phys. Rev. C 2007 75, 055207. [CrossRef]

19. Cebulla, C.; Goeke, K.; Ossmann, J.; Schweitzer, P. The Nucleon form-factors of the energy momentum tensor in the Skyrme model. Nucl. Phys. A 2007 794, 87. [CrossRef]

20. Balitsky, I.I.; Braun, V.M.; Kolesnichenko, A.V. $\Sigma^{+} \rightarrow P \gamma$ Decay in QCD. Sov. J. Nucl. Phys. 1986, 44, 1028.

21. Balitsky, I.I.; Braun, V.M.; Kolesnichenko, A.V. Radiative Decay Sigma $\rightarrow$ p gamma in Quantum Chromodynamics. Nucl. Phys. B 1989, 312, 509. [CrossRef]

22. Braun, V.M.; Lenz, A.; Wittmann, M. Nucleon Form Factors in QCD. Phys. Rev. D 2006, 73, 094019. [CrossRef]

23. Braun, V.M.; Lenz, A.; Mahnke, N.; Stein, E. Light cone sum rules for the nucleon form-factors. Phys. Rev. D 2002, 65, 074011. [CrossRef]

(C) 2019 by the author. Licensee MDPI, Basel, Switzerland. This article is an open access article distributed under the terms and conditions of the Creative Commons Attribution (CC BY) license (http://creativecommons.org/licenses/by/4.0/). 